\title{
Direct consultant supervision of higher trainees in child and adolescent psychiatry
}

\author{
A survey of expectations and practice
}

\author{
Stephen Kingsbury and Mark Allsopp
}

\begin{abstract}
Higher trainees in child and adolescent psychiatry and their consultant trainers reported high levels of compliance with existing JCHPT guidelines on the structure of direct supervision. There was a consensus about the ideal content of supervision sessions but dispartity about whether this is achieved in practice and over issues of responsibility for raising certain topic areas. These findings are discussed and some questions to be addressed by trainee and trainer at the outset of the supervision process are suggested.
\end{abstract}

The direct supervision of higher trainees by their consultant trainers, involving face to face discussion, is often seen as a cornerstone of professional development in child and adolescent psychiatry and is undoubtedly a strong formative influence on attitudes concerning the specialty and its practice. Many practitioners will have experienced in their own training great variation in the style and content of supervision and reflected on the impact of this. While some experiences of supervision may be inspiring, others may serve only to demoralise. The extent to which it is possible and desirable to set standards, monitor guidelines and provide training for the supervision process is uncertain.

In the United Kingdom the Joint Committee on Higher Psychiatric Training [JCHPT], published a handbook [1990] which describes requirements and standards about specialty training. A system of regular inspection of training schemes identifies the extent to which these are met. Bools \& Cottrell (1990) presented results of a survey of higher trainees in child and adolescent psychiatry which suggested that changes in training and in trainees' attitudes when compared to an earlier survey in 1979 [Garralda et al. 1983] had broadly followed JCHPT guidelines. The committee can therefore be seen to function as an important link in an interactive process of training and educational development.

The JCHPT handbook gives firm guidelines about the frequency and regularity of direct con- sultant supervision. Even so Bools \& Cottrell (1990) were concerned that $27 \%$ of respondents were receiving less than one hour of direct consultant supervision per week and that nearly $10 \%$ reported receiving no individual supervision at all. The guidelines recognise that the practice of supervision varies widely and leave room for flexibility to meet the individual needs of trainees at different stages of training. While this approach permits adaptation and creativity within the supervision process, it also allows for misunderstandings arising from disparities of perceived requirement, expectation and beliefs about the nature of the task. The content of sessions, permission and responsibility to raise certain issues, and the nature of the supervisory relationship may form the subject of the difficulties.

We present the results of a survey of the perceptions of consultant trainers and higher trainees in child and adolescent psychiatry in England and Wales concerning their practical experience of the direct supervision process in which they were involved and their ideal. Our aim was to attempt to identify areas of consensus and disparity of view between groups of trainers and trainees which may help to point a direction for the development of future standards.

\section{The study}

Clinical tutors for each of the higher training schemes in child and adolescent psychiatry in England and Wales were contacted and asked to identify for the purpose of the survey all trainees (full-time and part-time senior registrars and clinical lecturers) attached to their scheme together with their designated consultant trainers. All schemes approached took part and a total of 115 trainees and 117 trainers were identified. Each doctor was contact separately and asked to complete a postal questionnaire. An undertaking was given not to identify responses from any 
single trainer and trainee dyad nor those from any particular training scheme. Non responders were prompted once after an interval.

The questionnaire was in two sections; one asked for the respondents' views about supervision in higher training in an ideal setting, the other about what occurred in practice. Each section contained similar questions about three distinct areas.

(a) The structure of direct consultant supervision of higher trainees. The questions asked were about the length, frequency and composition of direct supervision settings, whether they occurred at a fixed regular time and whether a mechanism for systematic review of cases and topics is used.

(b) The content of direct supervision. Respondents were asked to indicate the percentage of the time available which was or should ideally be allocated to the discussion of nine given domains. These were:

(i) service management and medical politics

(ii) theoretical approaches to clinical management

(iii) practical management of cases or case load

(iv) supervision of a session with an individual patient or family

(v) career and training issues

(vi) research issues

(vii) personal guidance or anxiety containment

(viii) teaching and supervision of others

(ix) discussion of the supervision process itself.

(c) The nature of the supervisory relationship be tween trainer and trainee. For each of the nine domains above the respondent was asked to indicate on a seven-point equal-interval scale the balance of responsibility held by trainer and trainee for raising the topic in supervision. Using similar scales respondents were also asked to rate the extent to which what were described as 'transference and counter-transference issues' arising between trainer and trainee are or should ideally be discussed in supervision. An overall rating of satisfaction with the current supervision for themselves and the other involved was also requested.

In total $84(73 \%)$ trainees and $93(79 \%)$ consultant trainers replied, giving an overall response rate of $76 \%$.

\section{Findings}

\section{Structure}

There were no significant differences between trainers and trainees so group results will be presented in this section.
Time. $98.8 \%$ felt supervision should occur at a fixed regular time. For $81.4 \%$ this often happened in practice, for $10.7 \%$ this often did not happen and $7.8 \%$ had no fixed regular time established.

Frequency. $94.2 \%$ felt that this should be at least weekly and $80 \%$ said that this occurred; $20 \%$ reported that the interval between supervision sessions was a fortnight or longer.

Length. 95.8\% felt supervision should last at least one hour; $86.7 \%$ felt this duration was achieved.

Composition. The ideal composition was one trainer with one trainee in $91.8 \%$ responses and occurred in practice for $90 \%$ respondents.

Review. $16.1 \%$ felt that there should be a systematic process of case review for every session and $62.8 \%$ for some sessions, while $20.9 \%$ felt there should be no systematic process. In practice, systematic review occurred in every session for $16.3 \%$ respondents, in some sessions for $22.7 \%$ and never occurred in $60.6 \%$.

\section{Content}

There was broad consensus between trainees and trainers on the ideal time allocation for each issue and no significant differences emerged using rank sum tests. The mean percentage time ideally allocated for each issue by all respondents was:

(a) service management and medical politics $10.4 \%$

(b) theoretical approaches to clinical management $13.1 \%$

(c) practical management of cases or case load 28.6\%

(d) supervision of a session with an individual patient or family $15.3 \%$

(e) career and training issues $8.1 \%$

(f) research issues $8.8 \%$

(g) personal guidance or anxiety containment $5.9 \%$

(h) teaching and supervision of others $6.7 \%$

(i) discussion of the supervision process itself $4.1 \%$.

The percentage time allocations trainees reported in practice significantly differed from their ideal allocations for a number of issues. More time was reported spent in practice on the management of cases and case load (41.4\%) than was ideal (27.9\%; $P=0.0045)$ and less on discussion of theory (practical $10.0 \%$, ideal $12.9 \%$; $P<0.0001$ ), career issues (practical 6.5\%, ideal $8.4 \%$; $P=0.0047$ ) teaching (practical $3.6 \%$, ideal $7.5 \%$; $P=0.0003$ ) and the discussion of the supervision 
process (practical $1.3 \%$, ideal $4.7 \% ; P=0.0002$ ). In contrast, the trainers identified no significant differences between ideal and practical settings, although there was a trend to agreed that the time spent on case management issues was more than ideal (practical $35.0 \%$, ideal $29.2 \%$ ).

There were also significant differences between the perceptions of the trainees and trainers about the percentage time allocation to a number of issues in practice. Trainers felt that more time was spent on career issues (trainers $8.4 \%$, trainees $6.5 \% ; P=0.006$ ), personal guidance (trainers $5.3 \%$, trainees $3.7 \% P=0.001$ ), teaching (trainers $5.3 \%$, trainees $3.6 \% ; P=0.02$ ) and discussion of the supervision process (trainers $2.7 \%$, trainees $1.3 \% ; P=0.001$ ) than did trainees.

\section{Relationship}

Responsibility. As judged by the relationship of the overall mean rating to the mid-point (4) of the range of the interval scale $(0-7)$, there was broad consensus that in an ideal supervision setting trainers should be more responsible for initiating discussion of service issues (mean=3.6), theory (mean=3.5) and discussion of the supervision process (mean=3.6); that trainees should be more responsible for raising matters to do with personal guidance (mean $=4.7$ ) and individual case management (mean=4.4); and that responsibility for supervision of particular therapy sessions (mean=4.1), discussion of career (mean=4.0) and teaching issues (mean $=3.9$ ) should be equally shared. However, on three issues trainees thought that ideally they should carry more responsibility for initiating discussion than in the trainers' view they should. These were practical case management issues $(P=0.002)$, research issues, $(P=0.008)$ and personal guidance $(P=0.01)$.

The overall group means for the situation in practice suggest that more responsibility for initiating discussion rests with the trainee for many issues (case management mean $=4.8$, research issues mean $=4.5$, personal guidance mean $=4.5$, supervision of sessions mean $=4.3$, teaching mean=4.3, career mean=4.2). For others responsibility is equally shared. However, for each issue there was a significant difference between trainers' and trainees' ratings. Trainees' responses suggested that they feel more responsible in practice than the trainers believe them to be for initiating discussion of service issues $(P=0.01)$, theoretical issues $(P=0.0001)$, case management $(P=0.0001)$, career $(P=0.0003)$, research $(P=0.002)$, personal guidance $(P=0.0001)$, teaching $(P=0.0001)$ and the supervision process $(P=0.0005)$.

A comparison of the ratings of responsibility for initiating discussion in the ideal setting and in practice responses for each group demon- strated that trainers found no differences whereas trainees felt significantly more responsible in practice than the ideal for initiating discussion of theory $(P=0.0001)$, case management $(P=0.004)$, career issues $(P=0.004)$, teaching $(P=0.003)$ and the supervision process $(P=0.002)$.

Transference. There was a wide range in respondents' rating of the extent to which transference and countertransference issues should ideally be addressed in supervision and within the overall working relationship. On a seven-point equal interval scale where 0 was rated as not at all important' and 7 as 'very important' the overall mean for the supervision setting was 3.93 and for the relationship as a whole 4.12 . In practice, transference issues were felt to be attended to significantly less than ideally (supervision: mean 2.02, $P=0.0001$, relationship: mean 2.41 , $P=0.0001$ ). In addition trainees felt these issues were attended to in practice significantly less than trainers (supervision $P=0.0001$; relationship $P=0.0005$ ).

Satisfaction. $80.6 \%$ rated themselves as averagely satisfied or better (i.e. $19.4 \%$ rated themselves as dissatisfied to some degree) compared to $86 \%$ who rated the other party in supervision as satisfied to some degree. There were no significant differences between trainees and trainers.

Details of statistical information are available on request to Dr Allsopp.

\section{Comment}

This is the first survey to canvass the views of both trainees and trainers about the process of supervision in higher training in child and adolescent psychiatry and to enquire about aspects of content and the supervision relationship as well as organisational matters. Our aims were to explore the extent of consensus about direct training supervision in child and adolescent psychiatry, to see if current standards are met and whether pointers for the development of future standards emerged. The response rate was high so that the results are probably representative of the range of current opinion and practice.

There is a high degree of consensus among trainers and trainees about the ideal structure of supervision sessions and for upwards of $80 \%$ of trainees something approaching this ideal is met in practice. It is encouraging that the structure of supervision sessions preferred by over $90 \%$ of respondents in both ideal and practice, i.e. oneto-one weekly meetings, regularly timetabled and of at least one hour's duration, follows so closely that proposed by the JCHPT guidelines. Despite this, the study confirms the findings of Boots \& 
Cottrell (1990) that 10 to $20 \%$ of trainees have supervision experiences which do not meet these criteria. In contrast, there was considerable disparity between ideal and practice on the issue of regular review of cases. While $76 \%$ of respondents overall felt some form of review would be helpful, $60 \%$ reported using no mechanism in practice. There were no differences between trainees and trainers. Interestingly those advocating the use of systematic review in every session were more likely to be employing some form of review in practice.

Trainees and trainers largely agreed about the ideal percentage time allocated to most content areas, with review of cases being dominant. As a group, trainers perceived no significant differences between this ideal and current practice. The trainee group, however, reported significantly more time spent in case review than they felt ideal, largely at the expense of time spent on theoretical, career and teaching issues. In practice, differences in the perceptions of trainers and trainees were most marked in the areas of personal and career guidance.

Concerning responsibility to raise issues for discussion in supervision, there was broad consensus that trainers might be expected to take more initiative over service management and theoretical issues, while for case review and personal guidance trainees might take the lead, and for the other topic areas responsibility should be equally shared. Differences between trainer and trainee groups were all in the direction of trainees wishing to take more responsibility. Trainers' reports of practice did not differ from this ideal, whereas trainees felt that they carried more responsibility than the trainers realised and more than either group would perceive as ideal.

There was a wide range of opinion about the extent to which 'transference issues' between trainer and trainee should be discussed. In practice, discussion of this was significantly less than ideal, suggesting perhaps an awareness that some issues within the relationship between trainee and trainer are hard to address. The high rates of satisfaction reported are encouraging, but as no association with any other variables emerged, perhaps of limited value.

Thus there are several areas where, despite consensus about the ideal, there was a disparity between groups in perceptions of what occurs in actual practice. Unfortunately, it is not possible to evaluate which perception is the more accurate. It may be that trainers are correct in believing that practice follows the ideal and, if so, this would imply that trainees' perceptions are impaired by other aspects of the trainee role. Expectations and beliefs about hierarchical relationships and degree of autonomy in the light of their level of experience may be important. On the other hand, trainees may perceive the disparity accurately, implying perhaps that trainers are failing to recognise that they are not meeting their own ideals. This may be because they have insufficient time or opportunity to reflect critically upon the supervision which they do not have the resources to implement. Finally, the disparity may represent a mismatch of expectations and beliefs about the trainee role between trainee and trainer together with a failure to communicate about the issue. One striking finding from the survey was the small amount of time reported allocated to discussion of the supervision process itself. Trainees as a group felt that this small proportion was less than ideal, but for trainers ideal did not differ from practice.

In summary, there was a high level of consensus about what should ideally occur in direct supervision, but much less agreement that this happens in practice. This is particularly the case in the areas of systematic review, the preservation of time to discuss topics other than case management and the division of responsibility for raising issues in supervision. Time spent discussing the supervision process to clarify requirements and expectations might help to reduce misunderstandings and discrepancies in perceptions identified by the survey.

Finding the answers to a short checklist of questions even on an informal basis at the outset of the supervision relationship might be found useful. Such questions could be as follow.

(a) What will be the structure for our meetings?

(b) What systematic review of cases does the trainer wish?

(c) How shall we plan that over time all topic areas are covered (so that theoretical, career and teaching discussions are not left out)?

(d) Who will carry responsibility for raising each topic and issue?

(e) How and when shall we review the supervision process?

The great extent to which existing JCHPT guidelines are met may reflect the effectiveness of inspection in achieving an interactive process of educational development. It may be that the simplest way of extending guidelines and standards about supervision without impairing the flexibility and creativity which is highly valued would be to require that the answers to such a checklist be agreed between each trainer and trainee and be readily available at inspection visits.

\section{References}

Bools, C. \& CotTrell. D. (1990) Future child and adolescent psychiatrists: a further survey of senior registrar training. Psychiatric Bulletin, 14, 611-615. 
GARRALDA, M.E., Wiselberg, M. \& MRAZEK, D. (1983) A survey of training in child and adolescent psychiatry. British Journal of Psychiatry, 143, 498-504.

JoINT COMMTTEe ON HigheR PSYChIATRIC TRAINING (1990)

Handbook London: Royal College of Psychiatrists

Stephen Kingsbury, Consultant in Child and Adolescent Psychiatry, Queen Elizabeth II Hospital,
Welwyn Garden City, Hertfordshire AL7 4HQ; and "Mark Allsopp, Consultant in Child and Adolescent Psychiatry, Marlborough Childrens Hospital, The Common, Marlborough, Wiltshire, SN8 1JT

*Correspondence

\title{
Use of approval visits by the CTC*
}

\author{
Roger Bullock
}

Recent concerns have arisen at Collegiate Trainees Committee (CTC) meetings, the Central Approval Panel (CAP), and to a lesser extent the Joint Committee on Higher Psychiatric Training (JCHPT), which can be summarised thus:

(a) the continued lack of profile of the CTC in some regions

(b) low morale in trainees

(c) CAP convenors using non-CTC senior registrars for approval visits - creating a sense of 'in-house' decisions being made

(d) poor knowledge of trainees about what an approval visit means

(e) inadequate feedback to trainees after an approval visit and the impact of recommendations made

(f) lack of organised representative trainee bodies at scheme level.

These six problems are not unrelated and it is possible to draw them together and achieve a substantial improvement by using the structure of the CTC and its representation on both the CAP and JCHPT. This can be achieved by:

(a) taking advantage of their position on CAP and JCHPT. Representatives know when an approval visit is due and can

(i) notify the appropriate local CTC members of any visit in their division

(ii) be responsible for selecting a CTC member to be the trainee member of a visit - or if a CTC member is not available, assist the convenor to find a suitable replacement.

(b) Local CTC representatives should identify themselves to the local convenors

-This document has been accepted by the CTC/CAP and JCHPT. who can ask their assistance in finding members.

(c) Where possible, the local CTC representatives should visit the hospital/scheme in advance of a planned CAP/JCHPT inspection. They would use the meeting to:

(i) explain the function of the CTC

(ii) explain the function of the approval visit (using the CTC prepared leaflet)

(iii) emphasise the good working relationship that exists between trainees and tutors and ensure they are working together to improve training rather than acting in adversarial positions during an approval visit

(iv) inform trainees of what training and supervision they are entitled to and what their timetable should look like

(v) reassure trainees that their views will be taken seriously with confidentiality guaranteed

(vi) view the recommendations from the previous approval visit

(vii) strongly encourage trainees to attend the feedback session at the end of a visit ensuring that they hear the draft recommendations

(viii) explain the significance of the recommendations and the difference between mandatory and nonmandatory objectives, and what happens to the draft recommendations before they are passed by the College.

(d) Following an approval visit the local CTC representative should make it clear that 